\title{
Diagnostic des projets de réinsertion économique des migrants de retour : étude de cas au Mali (Bamako, Kayes)
}

Babacar Ndione et Jérôme Lombard

\section{(2) OpenEdition \\ Journals}

Édition électronique

URL : https://journals.openedition.org/remi/316

DOI : $10.4000 /$ remi.316

ISSN : $1777-5418$

Éditeur

Université de Poitiers

\section{Édition imprimée}

Date de publication : 1 mai 2004

Pagination : 169-195

ISBN : 2-911627-36-9

ISSN : 0765-0752

Référence électronique

Babacar Ndione et Jérôme Lombard, « Diagnostic des projets de réinsertion économique des migrants de retour : étude de cas au Mali (Bamako, Kayes) ", Revue européenne des migrations internationales [En ligne], vol. 20 - $\mathrm{n}^{\circ} 1$ | 2004, mis en ligne le 25 septembre 2008, consulté le 15 avril 2022. URL : http://journals.openedition.org/remi/316 ; DOI : https://doi.org/10.4000/remi.316

Ce document a été généré automatiquement le 15 avril 2022

(C) Université de Poitiers 


\title{
Diagnostic des projets de réinsertion économique des migrants de retour : étude de cas au Mali (Bamako, Kayes)
}

\author{
Babacar Ndione et Jérôme Lombard
}

1 Le retour en vue de la réinsertion dans le pays d'origine constitue pour beaucoup de migrants une étape importante du processus migratoire international. Il s'inscrit souvent dans le projet initial du migrant et est étroitement lié à une réinstallation réussie, elle-même subordonnée à une réinsertion économique productive durable. Il s'agit pour l'individu de mettre en œuvre un projet économique rentable qui lui permette, lui et sa famille, d'assurer leur bien-être, afin qu'à l'avenir, il (ou sa famille) ne ressente plus la nécessité d'émigrer à nouveau.

2 L'idéologie du projet collectif, initié au retour des migrants, est très développée dans certains pays d'Afrique de l'Ouest. Ce type de projet semble avoir beaucoup plus d'effets sur l'environnement territorial, social et économique que le projet mené par un individu ${ }^{1}$. Ces projets concernent un périmètre irrigué, un centre de santé ou bien une association de développement. Le regard porté sur les projets individuels est par conséquent souvent critique, voire inexistant. Or, ces migrants de retour, rentrés avec un projet, existent en grand nombre. Beaucoup d'entre eux prennent l'option de retourner vivre dans leur pays d'origine et de réaliser un projet d'investissement personnel. Le retour peut être autonome, à leur initiative et sans aucun soutien des bailleurs de fonds ou être opéré dans le cadre de programmes d'aide, développés soit par les organisations internationales, soit par le biais d'accords bilatéraux établis entre les pays d'accueil et les pays d'origine. Dans les deux cas, la mise en œuvre du projet détermine souvent le retour du migrant dans son pays d'origine.

On sait encore relativement peu de chose sur le devenir des projets de réinsertion déjà réalisés. À partir d'une étude menée en 2002 par l'OIM (Organisation Internationale pour les Migrations) et l'IRD, auprès d'une centaine d'émigrés maliens revenus à 
Bamako et dans la région de Kayes (Mali), il est possible d'évaluer la réussite des projets individuels de réinsertion ${ }^{2}$. L'avenir des analyses menées sur ces investissements est prometteur : d'une part, pour étudier et améliorer ce qui a déjà été fait; d'autre part, pour comprendre le rapport de la société à la migration, notamment ce qu'apporte ou signifie un projet économique soutenu, à distance ou sur place, par un individu.

Dans une première étape, nous essayons de donner un aperçu de la migration internationale de retour, en considérant les pays de dernière migration et les régions de résidence au Mali. Dans un second temps, nous nous intéressons aux projets de réinsertion économique développés, par les migrants de notre échantillon, dans le district de Bamako et dans la région de Kayes (98 individus). Dans cette optique, une première série de questions a trait aux caractéristiques socio-économiques et géographiques des émigrés et aux impacts sur l'évolution de chaque projet de réinsertion. Une deuxième série de questions concerne le parcours migratoire de l'individu et son influence sur le démarrage et l'évolution de chaque projet. Une troisième série de questions interroge l'évolution dans la durée des projets, en fonction de l'esprit d'entreprise des promoteurs, des capitaux investis, des difficultés rencontrées et des conditions dans lesquelles celles-ci ont été ou non surmontées.

\section{Les migrations internationales de retour au Mali}

Le Mali a la particularité d'être un pays de fortes dynamiques migratoires internationales. Ces déplacements se caractérisent par la diversité des lieux de destination, de transit, de ré-émigration, mais aussi de retour et de réinvestissements économiques et symboliques.

\section{Principaux pays de la dernière migration : la Côte d'Ivoire et la Mauritanie}

6 Les données du recensement de la population de 1998 au Mali $^{3}$ (carte 1) montrent la prépondérance des migrants de retour en provenance de la Côte d'Ivoire, l'un des principaux pays d'accueil des migrants internationaux maliens. En réalité, la Côte d'Ivoire occupe une place importante dans les flux migratoires internationaux des pays de la région ouest-africaine : la moitié des migrations internationales a ce pays soit pour origine, soit pour destination (REMUAO (Réseau Migrations et Urbanisation en Afrique de l'Ouest), 1998). Les flux migratoires entre le Mali et la Côte d'Ivoire représentent, selon cette même source, $12 \%$ du total des migrations internationales entre les sept pays ${ }^{4}$ du REMUAO au cours de la période $1988-1992^{5}$. Cette proportion est importante pour les échanges entre le Burkina Faso et la Côte d'Ivoire (40 \%) et faible pour les déplacements entre le Niger et la Côte d'Ivoire (5\%). Selon le recensement de 1998, le deuxième pays de provenance des Maliens est la Mauritanie, suivi par le Burkina Faso. 
Carte 1 : Migrants internationaux de retour selon le pays d'immigration et la région de résidence au Mali en 1998

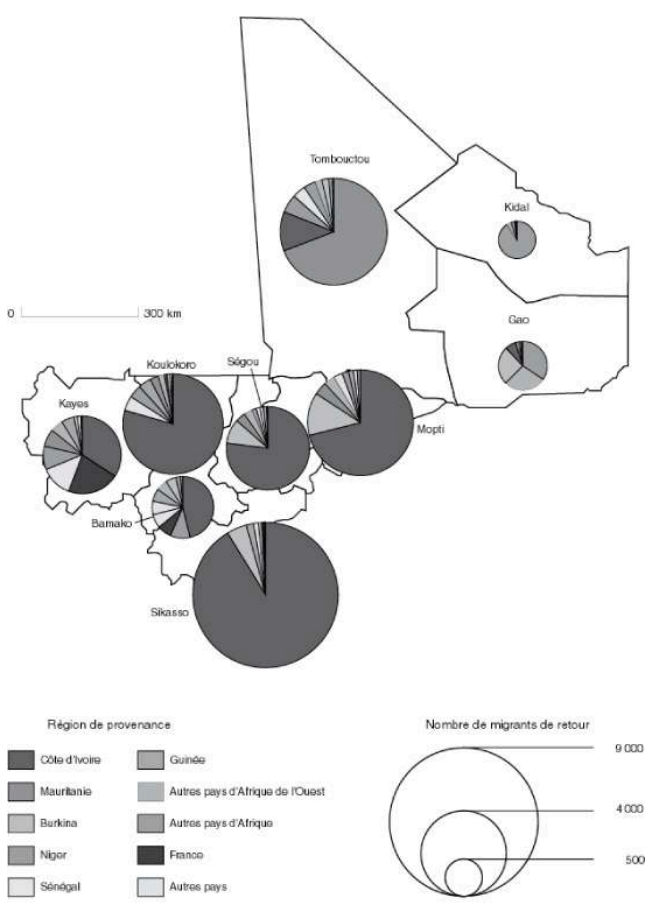

Réalisation : Axel Baillon, assistant à I'UMR IRD-OIM, Dakar, 2002. Source : RGPH, Mali, 1998.

7 La région ouest-africaine est le principal espace de migration internationale des Maliens : l'essentiel des individus de l'échantillon revient d'une migration des pays d'Afrique de l'Ouest (carte 1). Elle est suivie de loin par les autres pays de l'Afrique et les pays non africains. Cette dynamique migratoire s'explique par la longue tradition des mouvements de population dans la région. Avant l'époque coloniale, la région ouest-africaine était considérée comme une unité économique qui favorisait la circulation des biens, des services et des personnes. La domination coloniale a fortement contribué à alimenter de nouveaux courants migratoires de grande envergure. À l'indépendance, avec la préservation des frontières héritées de la colonisation qui délimitent de vastes ensembles géographiques, on aurait pu s'attendre à une réduction importante des déplacements. Au contraire, certaines frontières coloniales, défendues par les États indépendants, n'ont gardé qu'une signification théorique, séparant de part et d'autre des groupes ethniques qui constituaient avant un seul ensemble ${ }^{6}$. Ces frontières sont cependant devenues réelles au point que les nouveaux États, au cours des années, ont eu tendance à protéger leur territoire et à mener des politiques migratoires protectionnistes ${ }^{7}$. Celles-ci vont d'ailleurs aujourd'hui à l'encontre du principe de libre circulation des personnes qui a été adopté par les 15 pays membres de la Communauté Économique Des États de l'Afrique de l'Ouest $\left(\mathrm{CEDEAO}^{8}\right)$. Après les indépendances, les contraintes politiques n'ont pas empêché les échanges de populations d'augmenter entre les pays d'Afrique de l'Ouest, constituant ainsi une caractéristique principale de la dynamique de la population ouest-africaine. Cette relation migratoire prévaut encore entre le Mali et ses voisins. 


\section{Les régions de résidence des migrants internationaux de retour}

8 Toutes les régions du Mali accueillent des migrants internationaux de retour. L'importance du phénomène du retour varie considérablement d'une région à une autre. Le croisement des deux variables, région d'accueil du migrant de retour et pays de dernière destination, permet de mieux appréhender la dynamique migratoire de chaque région (carte 2 ).

Carte 2 : Migrants internationaux de retour selon la région de résidence au Mali et le dernier pays de destination

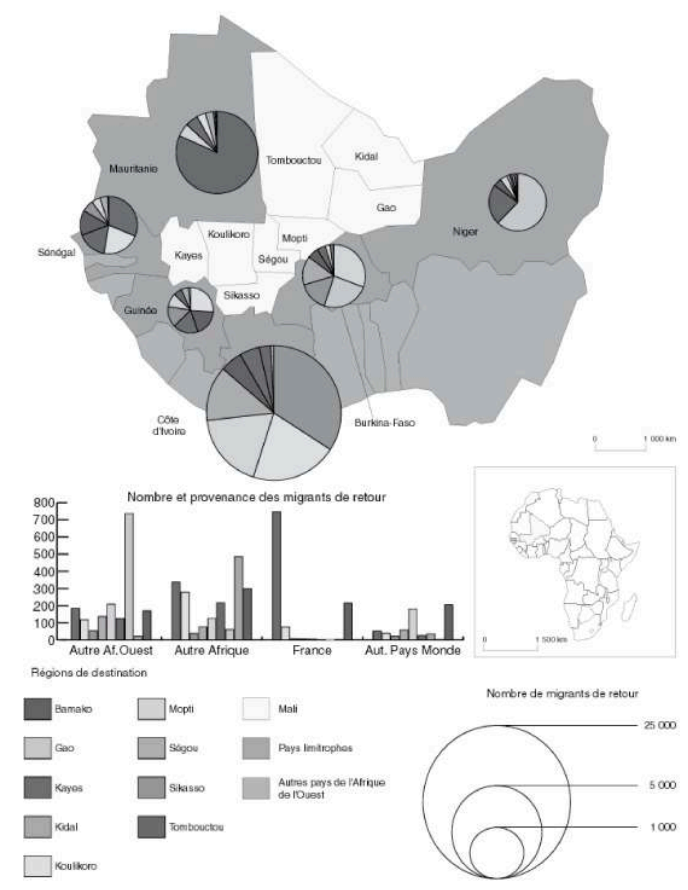

Réalisation : Axel Baillon, assistant à I'UMR IRD-OIM, Dakar, 2002.

Source : RGPH, Mali, 1998.

Les neuf régions du Mali ont une frontière avec un pays voisin et, de ce fait, alimentent la migration internationale de proximité, même si on sait que le lieu de retour ne correspond pas toujours au lieu d'origine, notamment pour les migrations vers le Nord. Toutefois on peut émettre l'hypothèse d'une forte migration transfrontalière qui expliquerait en grande partie la forte concentration des migrants de retour dans certaines régions. C'est le cas pour Sikasso dont $91 \%$ des migrants de retour établis proviennent de la Côte d'Ivoire limitrophe. Cette forte attraction de la Côte d'Ivoire concerne en réalité toutes les régions situées au sud et à l'ouest du Mali : Koulikoro (79\% des migrants de retour installés), Ségou (77\%), Mopti (71\%) et Kayes (34\%). En plus de la proximité de la Côte d'Ivoire, cette attraction s'explique aussi par le niveau de développement économique qu'a connu ce pays dans les années 1970-1980. La même relation migratoire de proximité est observée entre Tombouctou et la Mauritanie : $69 \%$ des migrants installés dans cette région reviennent de ce pays. Il en est de même pour Gao dont $34 \%$ et $25 \%$ des migrants de retour proviennent respectivement du Burkina 
Faso et du Niger. Ces tendances migratoires ouest-africaines confirment que les migrations sont fortement ancrées dans l'histoire des peuples de la sous-région.

Le retour en provenance des pays de l'Afrique de l'Ouest se double de mouvements d'origine extra-régionale et extra-continentale de moindre envergure, essentiellement engendrés à partir de l'Afrique centrale, de l'Afrique australe et de l'Europe (carte 2). Dans le cadre des retours d'une migration internationale lointaine, Kayes et Bamako sont les principales régions réceptrices avec, respectivement, $32 \%$ et $20 \%$ des effectifs. La prédominance de ces deux régions est particulièrement marquée pour les retours de France et des pays non africains. En ce qui concerne les pays de l'Afrique, cinq régions se distinguent: Kidal (région la moins réceptrice de migrants), Kayes, Bamako, Koulikoro et Tombouctou.

11 La région de Kayes appartient au bassin du fleuve Sénégal dont la particularité est d'être un foyer d'émigration internationale vers les pays du Nord, notamment vers la France (Kane et Lericollais, 1975 ; Condé et Diagne, 1986 ; Quiminal, 1991). Les migrants internationaux en général, et ceux de la région de Kayes en particulier, s'installent à leur retour dans leur région d'origine ou optent pour d'autres régions plus attractives, en fonction de leurs aspirations et des activités économiques qu'ils comptent développer. C'est dans cette optique qu'il faut considérer le choix de Bamako comme un lieu de retour important: en tant que capitale et principale ville du Mali, Bamako concentre la majorité des activités économiques et offre aux migrants des opportunités de réinsertion plus importantes que les autres régions du Mali ${ }^{9}$. Selon l'Enquête malienne de conjoncture économique et sociale (5), la région de Kayes polarise l'essentiel des flux de transferts monétaires des émigrés maliens (61\%), suivie de loin par Bamako et Ségou ( $9 \%$ chacune) et par Mopti et Tombouctou (6\%). Pour les régions de Kayes et Bamako, la destination des émigrés explique les différences enregistrées dans les montants des flux de transferts : «les envois de fonds en provenance d'Europe, et de France en particulier, sont beaucoup plus importants en volume que ceux en provenance d'autres pays d'Afrique de l'Ouest ${ }^{10}$.

12 Les régions de Kayes et Bamako ont ainsi été sélectionnées par notre étude afin de mieux rendre compte des investissements réalisés par les migrants de retour et d'éviter de concentrer l'étude sur les flux en Afrique de l'Ouest.

\section{Méthode}

Une enquête sur la migration internationale en milieu d'origine soulève deux difficultés. Par définition, la migration internationale est un événement rare au sens statistique du terme. Elle est soumise à un fort "effet de grappe »: les migrants internationaux de retour ne sont pas répartis uniformément sur l'ensemble d'un territoire donné ; ils témoignent au contraire d'une forte concentration géographique, à des échelles aussi grandes que le quartier, l'îlot urbain ou le village. La difficulté de repérage s'accroît quand il s'agit de cibler parmi eux un sous-groupe particulier, en l'occurrence les migrants qui, à leur retour, ont réalisé des projets de réinsertion économique.

14 En l'absence d'une base de sondage appropriée, une enquête de prospection s'est révélée nécessaire pour faciliter l'identification et la localisation des migrants de retour promoteurs de projet. Cette démarche, fondée sur la dynamique des relations 
interpersonnelles, devait permettre d'identifier des migrants de retour qui, une fois contactés, pouvaient servir de relais vers d'autres en provenance du même pays d'immigration ou de la même localité d'origine au Mali. Cette stratégie d'enquête entre les migrants suppose pour être efficiente :

- - que, les migrants originaires d'un même pays et vivant le plus souvent en communauté dans les pays d'accueil tissent des relations qui leur permettent de rester en contact au retour;

- - que les migrants originaires d'une même localité de départ se connaissent.

Selon cette méthodologie, nous avons dénombré 189 migrants internationaux promoteurs de projet à Bamako et à Kayes. À partir de cette liste, nous avons sélectionné un échantillon de 100 personnes à qui nous avons administré le questionnaire. Une des limites de cette approche est qu'elle peut exclure les migrants n'ayant pas ou peu de liens sociaux et économiques avec les autres migrants de retour.

Les 100 individus sélectionnés ont été répartis également entre les deux zones d'enquête : 50 individus à Bamako et 50 à Kayes. Les cinquante individus de chacune des deux zones d'enquête ont été tirés selon la méthode des quotas, afin de prendre en compte leur répartition selon l'activité économique exercée (type de projet), le pays de dernière destination (Afrique de l'Ouest, Afrique Centrale et Australe, Europe, Amérique, Asie), le milieu de résidence (urbain ou rural) et les modalités du retour (autonome ou dans le cadre d'un programme d'aide). Ce principe de tirage permet de répartir l'échantillon de façon proportionnelle à l'intérieur de toutes ces modalités et pour chacune de ces variables. En d'autres termes, nous avons recherché la diversité des situations la plus grande possible, tout en respectant le poids relatif de chacune d'entre elles. Il ne s'agit pas de dresser le portait de la population des migrants de retour porteurs d'un projet, mais de rendre compte des processus actifs mis en œuvre par cette catégorie de personnes pour réaliser un projet de réinsertion.

\section{Quels promoteurs et quels projets ?}

17 L'explication des réussites et échecs des projets de réinsertion économique des migrants de retour repose sur une analyse subjective telle que produite par les promoteurs eux-mêmes ${ }^{11}$. Une évaluation des projets demanderait une analyse technique, financière, économique et même sociale.

\section{Les secteurs d'investissements}

Les secteurs d'activités dans lesquels les migrants promoteurs ont investi sont divers : commerce (magasin, boutique de détail), agriculture et élevage (jardin maraîcher, embouche), transport, vente de service (moulin à mil, cabine de téléphone privé), artisanat, restauration. Le commerce domine (39\%): sous forme d'un magasin, d'un entrepôt dans lequel on trouve des céréales, du ciment, du matériel de construction, du matériel importé varié, etc. Une deuxième catégorie d'investissements concerne l'agriculture et l'élevage : embouche, jardin et matériel agricole (27\%). La majorité des émigrés promoteurs (notamment ceux de la région de Kayes) étant d'origine rurale, l'agriculture constitue un débouché, et parfois la seule possibilité, pour investir. Le transport apparaît en troisième position $(13 \%)$ : cela se matérialise, soit par la réfection d'un véhicule acquis pendant la période migratoire, soit par l'achat d'une automobile 
au retour. Les promoteurs se lancent alors, soit dans le transport de voyageurs (interurbain ou urbain), soit dans le transport de marchandises (camion benne notamment). Dans ces sociétés et économies fonctionnant sur la mobilité des personnes et des biens, et avec l'importance de l'urbanisation, le développement d'une activité de transport est opportun ${ }^{12}$, même s'il s'agit d'une activité dans laquelle le migrant rencontre beaucoup de difficultés, faute d'expérience. Dans l'artisanat, la restauration, la téléphonie, les promoteurs sont peu nombreux en raison de la compétence requise, des investissements que ces activités occasionnent et de la rentabilité incertaine qu'ils procurent.

Tableau 1 : Réussite ou échec des projets selon le type d'investissement économique avant retour

\begin{tabular}{|l|l|l|l|l|}
\hline Projet & Réussite & Echec & Ni l'un ni l'autre & Total \\
\hline Embouche & 13 & 6 & 6 & 25 \\
\hline Magasin de commerce & 4 & 1 & 2 & 7 \\
\hline Véhicule & 1 & 1 & 2 & 4 \\
\hline Équipement agricole & 2 & 1 & 1 & 4 \\
\hline Propriétés immobilières & 4 & - & - & 4 \\
\hline Boutique de détail & 1 & 2 & - & 3 \\
\hline Exploitation industrielle & 2 & - & - & 2 \\
\hline Moulin à céréales & 1 & - & - & 1 \\
\hline autres services & 1 & - & - & 1 \\
\hline Total & 29 & 11 & 11 & 51 \\
\hline
\end{tabular}

Source : Enquêtes 2002.

19 Comme on le note avec le tableau 1 , les migrants évaluent très diversement la réussite de leur projet. Sur les 33 magasins de commerce développés comme activité économique de réinsertion, $54 \%$ des projets sont considérés par leurs promoteurs comme une réussite. Les échecs sont ici relativement faibles. Dans les autres secteurs d'activités, c'est la situation inverse qui prévaut, puisque les migrants dressent généralement un bilan pour le moins mitigé, voir même franchement négatif. En moyenne, plus de la moitié des projets dans les secteurs suivants n'a pas fonctionné ou connaît des difficultés : boutiques de détail, transport, moulins à céréales, embouche, jardins maraîchers, équipements agricoles. Quant au secteur des télécommunications, il semble peu porteur car les trois projets développés (télécentre/cyber-informatique) ont échoué13. 


\section{Le processus de montage du projet est déterminant}

La personne qui initie le projet semble avoir un rôle déterminant dans sa réussite ou son échec. Plus de la moitié des promoteurs qui ont développé un projet de leur propre initiative a réussi sa réinsertion économique (graphique 1). Parmi les migrants ayant investi sur le conseil d'une tierce personne, $45 \%$ ont au contraire arrêté.

Graphique 1 : Réussite ou échec selon l'initiative de création du projet (en \%)

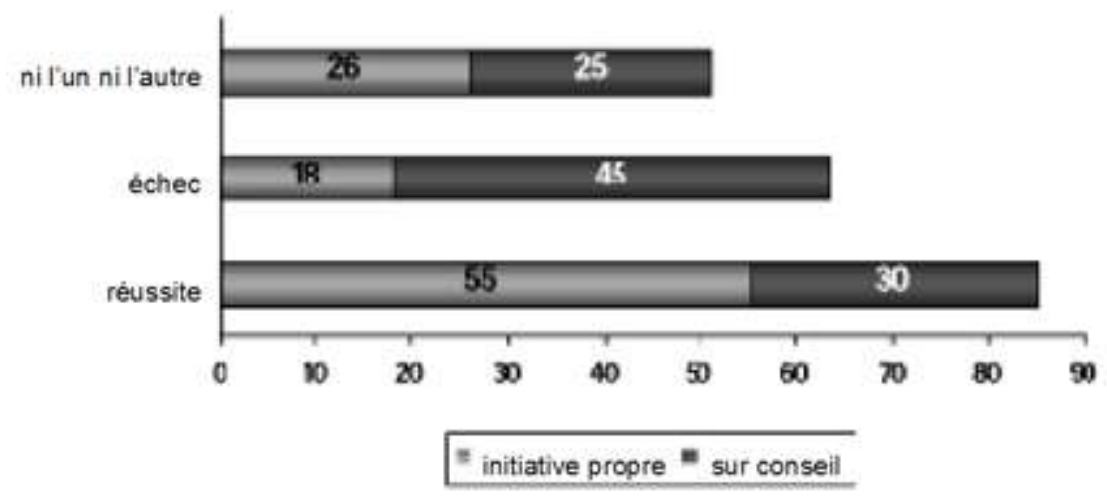

L'expérience dans l'activité développée est primordiale (graphique 2). $40 \%$ des promoteurs ont choisi leur secteur d'activité par opportunité, parfois un peu malgré eux. Par contre, $59 \%$ des migrants ont relancé une activité qu'ils avaient délaissée avant de partir en migration. Chez ceux-là, la proportion de réussite est la plus élevée.

Graphique 2 : Réussite ou échec selon la nature du processus de montage des projets (en \%)

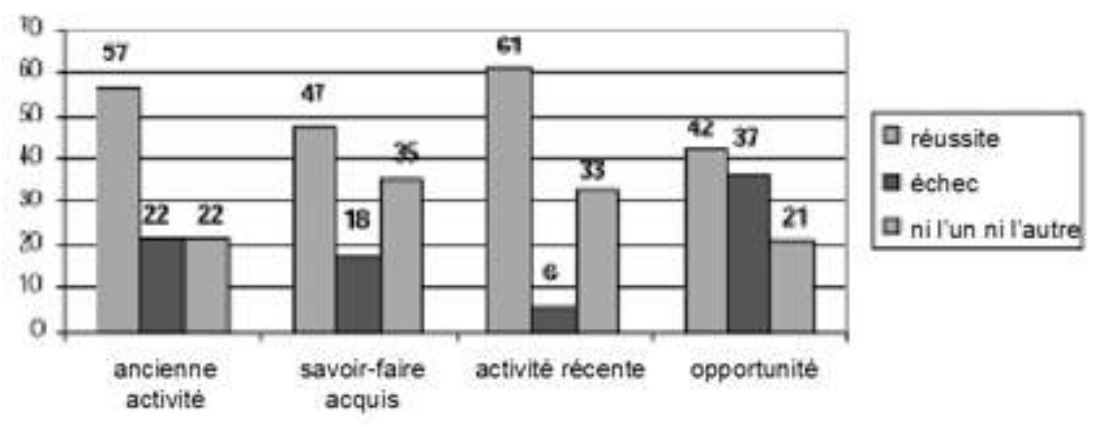

\section{Des investissements insuffisants}

L'analyse des sommes engagées dans les projets est assez difficile à mener, car les informations obtenues auprès des promoteurs sont incomplètes et disparates. $60 \%$ des promoteurs ont développé des projets de réinsertion d'une valeur totale de deux millions et plus (comprenant leur apport personnel). $24 \%$ ont investi moins de 1 million. Nombre de projets du type PDLM (Programme de Développement Local et Migration) ${ }^{14}$, en incluant les fonds personnels avaient ainsi une valeur d'investissement située loin en dessous de la dotation maximale (et souvent espérée des 2,4 millions).

Les investissements dans les projets autonomes, c'est-à-dire non aidés, ont été plus massifs que dans les projets aidés. La migration vers l'Afrique centrale a permis aux émigrés d'économiser d'importantes sommes susceptibles d'être investies dans la 
réinsertion économique. En outre, eux n'ont bénéficié de l'aide d'aucun bailleur de fonds, contrairement aux promoteurs émigrés en France qui, informés des programmes d'aides, ont souvent misé sur un soutien financier pour le développement de leur projet.

Plus des deux tiers des promoteurs (69\%) ont investi leurs économies dans le projet. Cette donnée est importante car elle va à l'encontre de l'opinion généralement répandue selon laquelle la réinsertion économique n'est pas possible sans aide extérieure. Trois éléments d'analyse sont à signaler. D'une part, la migration a permis à un certain nombre d'émigrés de réaliser une épargne et de démarrer un projet économique au retour. D'autre part, on observe que les projets nécessitent presque toujours un investissement de départ assez lourd; un projet sous-financé étant rapidement compromis en termes de développement et de rentabilité. Enfin, les aides semblent dans certains cas ne pas suffire.

Toutes les migrations ne permettent pas d'épargner, ou du moins pas au même niveau. L'éventail des montants investis signale qu'il y a des parcours migratoires, des pays d'accueil, des professions exercées à l'étranger, des processus de retour, des réinsertions locales, très différents les uns des autres et, qu'en aucun cas, on ne peut assimiler un émigré à un autre, un promoteur à un autre. Autrement dit, un émigré ne peut être considéré indépendamment de son origine familiale, de son contexte économique, social et géographique, de l'ensemble de son parcours migratoire : ce sont autant d'éléments qui peuvent expliquer la réussite ou l'échec du projet de réinsertion économique.

\section{Retour dans le milieu d'origine ou émancipation?}

La ville (Bamako et Kayes) est le milieu de réinstallation de près de $60 \%$ des promoteurs de l'échantillon. Proportionnellement, les projets qui ont réussi n'y sont pas plus nombreux qu'en milieu rural. Cependant, la ville ouvre des perspectives de développement plus importantes et plus intéressantes ${ }^{15}$, même pour un projet en difficulté. Ainsi, les migrants de retour sont plus nombreux en ville qu'en milieu rural à avoir une opinion mitigée sur la réussite de leur projet (ni échec, ni réussite). Les projets ressentis clairement comme des échecs sont proportionnellement plus importants en milieu rural (29\%).

\section{La famille à charge pèse-t-elle sur la réussite du projet ?}

L'analyse des réussites et échecs des projets (graphique 3) montre que c'est parmi les migrants ayant une charge familiale élevée (plus de 10 personnes) que l'on trouve la plus importante proportion de projets réussis. L'appartenance à un ménage de grande taille représente une situation favorable au développement des projets économiques, dans la mesure où le promoteur peut recourir à une aide familiale nombreuse et peu coûteuse. Inversement, c'est chez les migrants ayant à charge moins de 10 personnes que l'on trouve le plus grand pourcentage d'échecs. Les petits ménages (avec de jeunes enfants) ne sont pas d'un grand secours pour un promoteur confronté à des difficultés et qui souhaite réaliser des économies en s'appuyant sur la main-d'œuvre familiale (chaque promoteur concerné le dit : « ils mangent, mais ne m'aident pas ») ${ }^{16}$. 


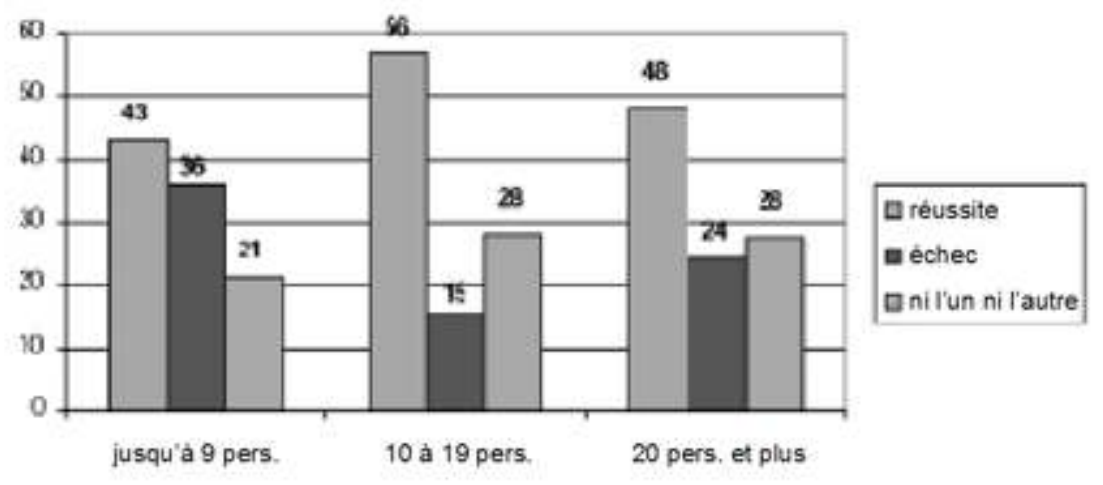

\section{Formation et expérience sur « le tas »} connaissance pratique du secteur d'activité, l'expérience professionnelle, l'importance du capital de départ et le soutien de la famille. Sans ces éléments critiques, la réussite du projet de réinsertion devient aléatoire. Le milieu urbain (Kayes, mais surtout le District de Bamako) constitue un lieu favorable au bon développement des projets économiques, y compris pour les migrants originaires de régions rurales. L'expérience urbaine des migrants, acquise pendant leur séjour à l'étranger, leur 
permet de mobiliser, au retour et plus facilement, les ressources sociales et économiques qu'offre la ville d'installation.

\section{Émigration et projet d'investissement}

Le processus d'insertion du migrant de retour relève de plusieurs paramètres qu'il faut replacer dans les contextes économiques et politiques des pays de destination et de départ, et à l'intérieur des relations privilégiées que le migrant entretient avec sa famille et sa communauté locale d'origine. L'émigration internationale a avant tout pour finalité de satisfaire les besoins monétaires de la famille restée dans le pays d'origine. La stratégie économique du migrant est donc tournée vers le milieu d'origine (famille et communauté). De façon beaucoup plus secondaire et plus tardive, la migration peut devenir aussi une stratégie d'enrichissement destinée à réaliser des investissements productifs dans le milieu d'origine.

\section{Le retour vu de l'étranger : transferts à distance et développement de projets futurs}

Dans le pays d'immigration, l'individu prépare-t-il sa réinsertion dans son pays d'origine, en prenant les dispositions nécessaires, notamment par des investissements économiques? Habituellement, le revenu de l'émigré est partagé entre ses dépenses personnelles dans le pays d'installation, les envois de fonds destinés à la famille et à la communauté locale d'origine et l'épargne constituée sur place ou thésaurisée au pays. D'après les enquêtes, les transferts annuels des émigrés sont destinés pour les deux tiers aux besoins domestiques de la famille d'origine, alors que la part consacrée à l'investissement dans un projet économique n'est que de $14 \%$.

L'utilisation de l'argent transféré est significative de stratégies familiales de survie, puisqu'une grande partie des dépenses de base est réalisée à la suite des transferts monétaires effectués par les émigrés. Ceux-ci servent en priorité à la couverture des besoins fondamentaux de la famille (alimentation, habillement et frais de santé). L'investissement immobilier capte aussi une partie de l'argent des émigrés (12\%). Les actions de solidarité envers l'entourage social du migrant et la communauté d'origine absorbent enfin une part non négligeable des transferts de fonds en faveur du pays d'origine ( $22 \%$ des transferts annuels). Regroupés en association villageoise ou de quartier (Daum, 1998), parfois au sein d'organisations régionales, les émigrés mobilisent d'importantes sommes d'argent pour les investissements sociaux au sein de leur localité d'origine.

La prise en charge de la famille et la réalisation des infrastructures communautaires sont prioritaires et passent avant tout autre action du migrant. Une fois ces deux obligations remplies, le migrant peut envisager un élargissement de son domaine d'intervention à des projets productifs. L'élevage - considéré traditionnellement comme une forme d'épargne notamment par les Haalpuularen - est un secteur d'investissement prédominant, puisqu'il capte $49 \%$ des transferts de fonds destinés au développement d'une activité économique productive. Les autres projets économiques développés à distance, même peu nombreux, contribuent aussi à préparer le migrant à sa réinsertion économique dans son pays d'origine : magasin de commerce (14\%), achat 
de véhicule de transport (8\%), équipement agricole (8\%), propriété immobilière (8\%), boutique de détail, exploitation industrielle, moulin à céréales etc.

Le démarrage en cours de migration d'une activité productive au pays influe sur la réussite de cette activité après le retour. Ainsi, plus de la moitié (57\%) des émigrés (tableau 2) ayant démarré une activité économique productive avant le retour a réussi son projet de réinsertion. Pour les autres, onze projets démarrés antérieurement au retour ont échoué et onze autres sont jugés en difficulté.

Tableau 2 : Réussite et échec selon le type de projet initié au retour de la migration

\begin{tabular}{|l|l|l|l|l|}
\hline Projet & Réussite & Echec & En difficulté & Total \\
\hline Magasin de commerce & 18 & 3 & 12 & 33 \\
\hline Véhicule de transport & 4 & 6 & 2 & 12 \\
\hline Embouche ovine & 5 & 2 & 2 & 9 \\
\hline Jardin maraîcher & 4 & 3 & 2 & 9 \\
\hline Equipement agricole & 5 & 1 & 3 & 9 \\
\hline Atelier artisanal & 4 & 1 & 2 & 7 \\
\hline \hline Boutique de détaillant & 2 & 3 & - & 5 \\
\hline \hline Société & 3 & 1 & - & 4 \\
\hline Moulin à céréales & 2 & - & 1 & 3 \\
\hline Télécentre/cyber & - & 2 & 1 & 3 \\
\hline \hline Restaurant & 1 & - & - & 1 \\
\hline \hline Vente carburant & - & 1 & - & 96 \\
\hline \hline Total & $\mathbf{4 8}$ & $\mathbf{2 3}$ & $\mathbf{2 5}$ & \\
\hline
\end{tabular}

Source : Enquêtes 2002.

\section{Des conditions de séjour à l'étranger, déterminantes pour le succès des projets}

37 La préparation du retour et de la réinsertion économique dépend de la situation sociale et économique de chaque migrant dans son dernier pays d'accueil (emploi, secteur d'activité, statut d'immigré, vécu quotidien et motif du retour). Ainsi que nous l'indiquions précédemment, Kayes et Bamako ont été retenues, car ces deux régions permettent de diversifier les parcours migratoires, en tenant compte à la fois des migrants partis dans les pays du Nord (France, notamment) et de ceux ayant vécu dans 
les pays africains. De ce point de vue, l'échantillon des 98 individus enquêtés est très significatif : $60 \%$ des émigrés ont été en Europe (France: $55 \%$ ). L'importance des personnes originaires de la région de Kayes, habituellement pourvoyeuse d'émigrés vers la France, explique cette forte proportion. Mais notre échantillon est constitué aussi à $38 \%$ de promoteurs de projet de retour d'un pays d'Afrique ( $28 \%$ en Afrique centrale et seulement $10 \%$ en Afrique de l'Ouest). Cette variété des parcours migratoires devrait rejaillir sur la nature des projets et leur mise en œuvre : activité, financement, réussite, difficultés, etc. Les conditions du retour et de l'émergence de l'idée de projet sont en effet liées en partie au pays dans lequel le migrant a passé ses dernières années de migration.

Contrairement à ce que nous pouvions attendre, les promoteurs en provenance d'Europe ne sont pas plus nombreux à juger positivement leur projet de réinsertion que les Maliens venant d'un pays d'Afrique (graphique 4). Les Maliens qui ont migré en France ont souvent bénéficié des financements accordés par la France; ils ont aussi acquis des expériences et des compétences qui leur permettent de mobiliser efficacement les ressources nationales, notamment en ville. Cependant, ces atouts ne semblent pas suffisants pour garantir la réussite du projet de réinsertion. Leur manque de formation et d'expérience dans l'activité qu'ils ont entreprise, ainsi qu'une préparation insuffisante des projets - parfois mal adaptés au contexte local demeurent un handicap important. En revanche, les migrants revenant d'Afrique, et notamment d'Afrique centrale, ont développé dans leur milieu d'origine un projet en rapport avec l'activité qu'ils exerçaient déjà en migration, c'est-à-dire le petit commerce. Ils sont près de la moitié à se déclarer satisfaits de leur projet. Même ceux qui jugent la réussite de leur projet médiocre sont mesurés : cette attitude propre aux commerçants doit être interprétée comme prudente et peu significative des réelles difficultés ou réussites.

Graphique 4 : Réussite ou échec selon le dernier pays d'accueil (en \%)

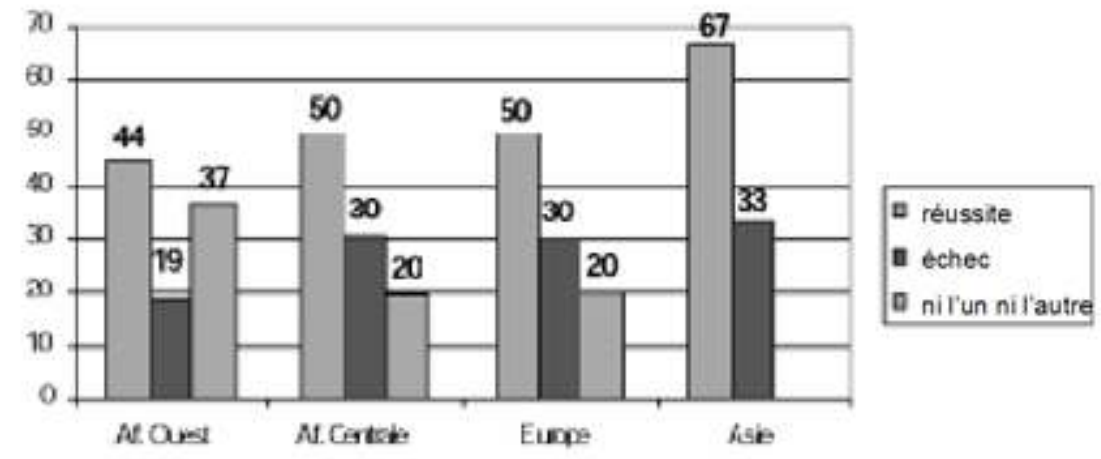

\section{L'activité rémunérée à l'étranger est indispensable}

En migration, la majorité des émigrés exerçait un métier peu qualifié : ouvriers spécialisés, manœuvres, employés de commerce. Dans ces secteurs d'activité, l'emploi est particulièrement précaire, expliquant sans doute que parmi les promoteurs interrogés, 14 \% étaient au chômage juste avant leur retour. Près d'un tiers des émigrés $(29 \%)$ avait une profession à plus haute qualification et responsabilité : employé du tertiaire, technicien, propriétaire d'un commerce. Les services, dont surtout la 
restauration, et le commerce sont les secteurs d'activité principaux des Maliens à l'étranger. Seuls $9 \%$ d'entre eux opéraient dans l'artisanat, secteur dans lequel les immigrés ont pu acquérir de nouvelles compétences, qu'ils ont parfois valorisées au retour.

Le fait d'avoir occupé un emploi rémunéré dans le pays d'immigration semble associé à la réussite d'une activité économique de réinsertion au retour (graphique 5). Les pourcentages de réussite sont en effet plus élevés chez les promoteurs ayant exercé, durant leur dernière année de migration, une activité économique rémunérée (52\%) que chez ceux qui n'ont pas travaillé (33\%). Les actifs enregistrent également le plus faible pourcentage d'échecs. Pour développer un projet de réinsertion, le capital initial d'investissement, accumulé grâce à un emploi rémunéré, paraît donc indispensable.

Graphique 5 : Réussite ou échec selon l'exercice d'un emploi dans le dernier pays d'accueil avant retour (en \%)

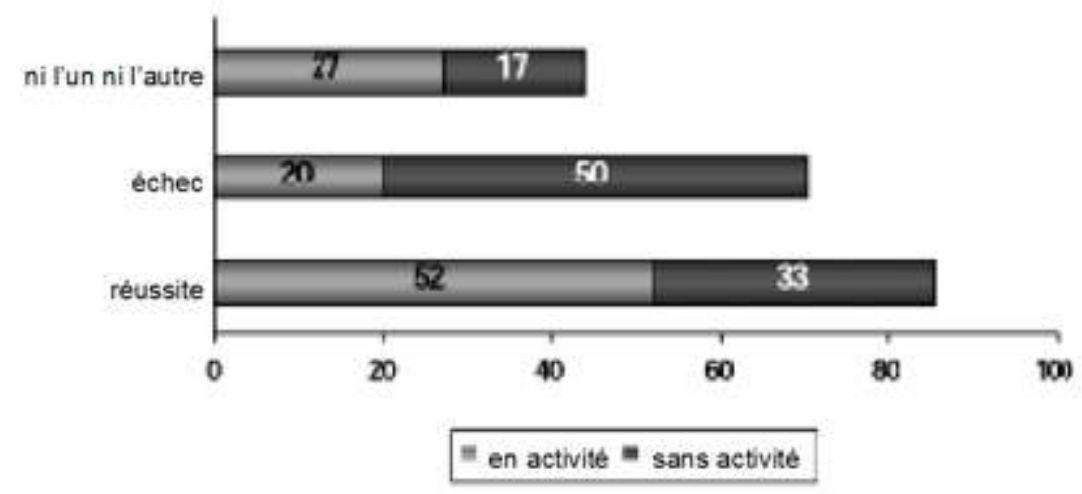

\section{Un retour motivé prépare à la réinsertion économique}

otifs de retour, tels que déclarés par les migrants, participent assez souvent d'un discours construit sur des arguments, en partie réels, mais qui sont toujours formulés pour préserver son image et sa position au sein de la communauté. Reste que même s'ils sont exprimés de façon plutôt favorable, ces motifs demeurent révélateurs des conditions difficiles dans lesquelles les migrants se trouvent au moment d'envisager un projet de retour. Un quart des émigrés $(24 \%)$ est rentré parce que le séjour à l'étranger (parfois leurs allées et venues) était « arrivé à son terme ", signifiant ainsi qu'ils étaient las de voyager ou d'être éloignés de la famille. Par ailleurs, 12 \% d'émigrés, en situation irrégulière, sont rentrés parce que le pays d'accueil (notamment la France) les a " poussés dehors ${ }^{18}$. Enfin, un autre quart des émigrés (22\%) a quitté le dernier pays de résidence à l'étranger en raison de troubles politiques ou de guerres civiles. Il s'agit essentiellement des Maliens installés en Afrique centrale, région traversée par de nombreux conflits depuis ces dix dernières années (les deux Congo, Angola, Burundi, Rwanda, etc.).

Les raisons familiales ou de santé sont des motifs de retour pour $24 \%$ des émigrés. La nécessité de revenir au village, pour répondre, souvent dans l'urgence, à ses responsabilités familiales ou à un problème de santé, sont souvent des retours réalisés sans préparation ni projet. À l'inverse, les $9 \%$ d'émigrés qui sont rentrés à la fin de leur contrat (retraite notamment) ou de leurs études (généralement en Europe) sont dans de 
meilleures conditions pour préparer une réinstallation au pays, au travers notamment d'un projet.

Les retours pour raisons familiales ou dus au rapatriement des migrants en situation irrégulière (graphique 6) semblent jouer négativement sur la réussite des projets : c'est seulement pour ces deux motifs de retour que l'on observe des pourcentages d'échec supérieurs à ceux des projets réussis. En fait parmi les retours précipités, seuls ceux qui sont survenus à la suite de troubles politiques dans les pays d'accueil ne sont pas associés à une plus forte proportion d'échec. Mais en dehors de cette exception notable, c'est bien évidemment parmi les migrants qui ont décidé, sans aucune contrainte, de rentrer vivre dans leur pays d'origine que l'on trouve le pourcentage de réussite le plus élevé. Ces émigrés ont eu le temps de préparer leur retour, soit en accumulant beaucoup d'argent pour investir au retour, soit en initiant précocement un projet économique à partir du pays d'accueil et, une fois sur place, le développer. Cette préparation n'évite pas les difficultés au moment de l'implantation du projet, mais elle semble en réduire les risques d'échec ${ }^{19}$.

Graphique 6 : Réussite ou échec selon le motif principal du retour (en \%)

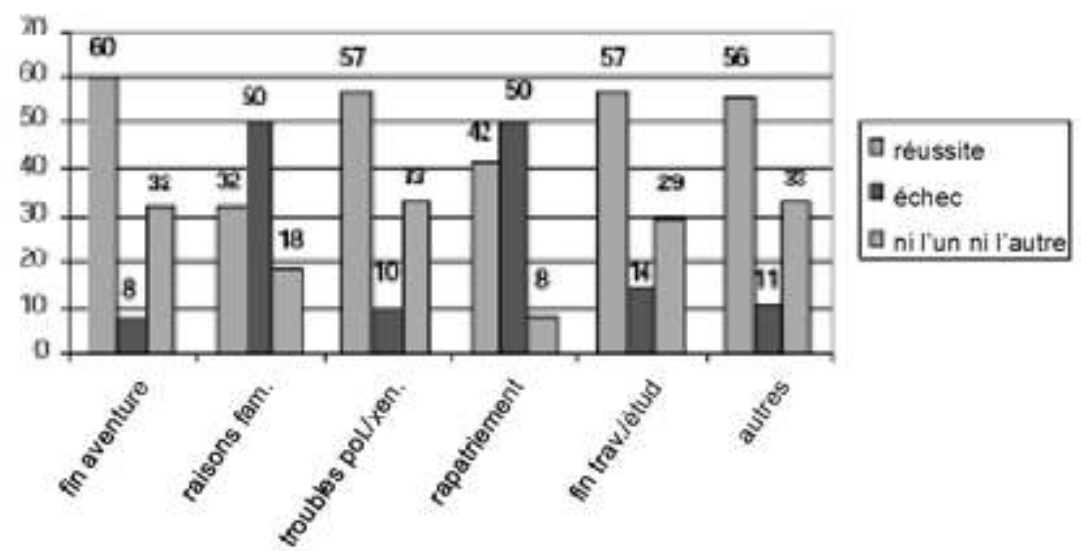

44 Le statut professionnel et social dans le dernier pays d'accueil du migrant est, beaucoup plus que le dernier pays de résidence, déterminant dans la réussite ou l'échec d'un projet de réinsertion économique. L'individu ayant un emploi rémunéré (dans les professions intermédiaires du commerce, des transports et des services) est prédisposé à développer un projet économique et à le réussir. En outre, ceux qui développent avant le retour un projet économique se mettent dans de bonnes conditions pour réussir leur réinsertion économique ; conditions d'autant plus favorables que le retour aura été réalisé de façon volontaire et sans contrainte.

\section{Réussite, difficulté ou échec : un promoteur n'égale pas un autre}

Les éléments expliquant la réussite, les difficultés ou l'échec des projets de réinsertion des migrants de retour sont à la fois multiples et variés. Ils dépendent de la situation individuelle de chaque promoteur, de son savoir-faire et de son environnement économique et social propre. 
Près de la moitié ( $49 \%$ ) des émigrés promoteurs pense que leur projet est aujourd'hui une réussite (graphique 7). Aux yeux des promoteurs, la réussite signifie que le projet permet de faire vivre au pays le migrant et sa famille. La réussite du projet s'exprime aussi par ses bénéfices ou des possibilités d'épargne, et ouvre enfin des perspectives d'expansion de l'activité («le chiffre d'affaires augmente au fil des années et permet une augmentation du capital investi »). Parfois, les émigrés jugent leur projet réussi, car il laisse entrevoir la possibilité de développer, sur place, d'autres projets économiques («complémentaires ») ou sociaux (« achat de terrain, construction »).

Graphique 7 : Succès ou échec des projets selon le jugement porté par les promoteurs (en \%)
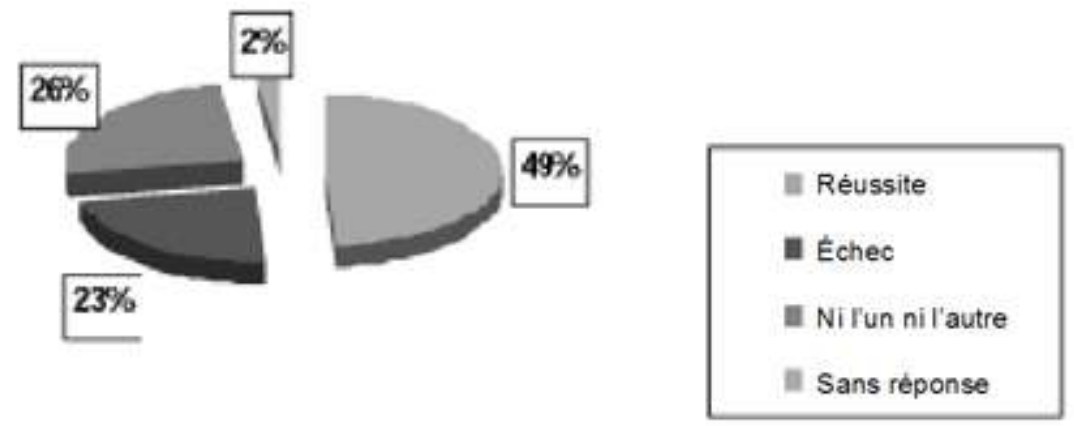

Inversement, les promoteurs qui ont échoué $(23 \%)$ indiquent que le projet n'a ni dégagé de bénéfices, ni contribué à améliorer l'ordinaire de la famille (en particulier dans les projets agricoles, d'élevage, de jardin). L'ambition de développer de nouveaux projets n'a pu être menée à son terme au point que certains émigrés sont repartis à l'étranger ${ }^{20}$. Parfois, les projets ont tout simplement été interrompus, sans possibilité de récupérer le capital investi.

Enfin, un quart des promoteurs ( $26 \%$ ) est insatisfait et estime que le projet n'est ni une réussite, ni un échec. Leur projet, même s'il rapporte faiblement, permet néanmoins la prise en charge des besoins fondamentaux de la famille. Certains émigrés se disent même optimistes quant aux possibilités de développement du projet. Ainsi, $13 \%$ des promoteurs concernés estiment que les difficultés ne sont pas rédhibitoires et correspondent à des problèmes de gestion (du temps, de la main-d'œuvre). Des solutions locales constituent alors la porte de sortie : augmenter le travail, recreuser le puits, clôturer le jardin, abaisser les taxes, trouver des pièces de rechange, si possible avoir de meilleurs hivernages, etc. Cependant, un peu moins de la moitié de ces promoteurs ( $45 \%)$ estiment que les difficultés rencontrées sont insolubles ou que la solution n'est pas à leur portée. Ils sont ainsi $18 \%$ à penser n'avoir aucune issue et ont démissionné. Plus d'un quart de ces promoteurs (27\%) considère que d'autres financements sont nécessaires pour relancer le projet.

\section{Quelle réussite pour quels projets?}

Les migrants qui ont réussi leur projet de réinsertion économique ont fait preuve de dynamisme aussi bien dans le développement de leur activité que dans la gestion de leurs affaires. L'esprit d'entreprise conditionne avant toute chose la bonne marche des affaires. 
bonne connaissance du secteur choisi influence positivement la réussite du projet de réinsertion : soit parce que l'individu a exercé le même métier avant ou au cours du processus migratoire (par expérience), soit parce que le promoteur jouit d'une compétence technique dans ce domaine (par formation professionnelle). Un projet économique demande au migrant une préparation minutieuse, aussi bien technique que financière. Ces dispositions doivent être accompagnées de connaissances en matière de gestion de projet pour permettre au promoteur de procéder régulièrement à l'établissement d'un bilan de ses activités. Afin de prendre les décisions et de mener à bien les actions qui concourent au développement de son projet, il lui est nécessaire de distinguer les coûts supportés par le projet, le chiffre d'affaires réalisé, le fonds de roulement de l'activité économique, le bénéfice réalisé, les charges liées à l'exploitation $\mathrm{du}$ projet, les dépenses de prise en charge de la famille (qui doivent représenter une partie du bénéfice réalisé et non la totalité de ce dernier), l'amortissement des équipements d'exploitation.

51 Pour réussir un projet économique, l'entrepreneur doit, dans le secteur de réinsertion choisi, entretenir des relations professionnelles. Ce qui fait marcher les affaires, ce sont les réseaux d'information sur les créneaux de développement de l'activité, les opportunités par secteur et par produit, les stratégies à développer pour s'approvisionner en marchandises, en produits de base ou en matières premières d'exploitation, les stratégies d'écoulement ou de vente des produits.

L'exemple d'un promoteur de jardin maraîcher, installé à proximité de Kayes, est très éclairant. Sur une parcelle héritée de son père, il a démarré un jardin dans son village natal dès son retour de l'étranger. L'argent du PDLM lui permet de développer son projet, par l'achat de grillages et de motopompes. Progressivement, le jardin devient aussi un lieu d'élevage et de production irriguée de maïs pour la vente au marché de Kayes. Parallèlement, ayant appris dans sa jeunesse la menuiserie avec son père, il monte un atelier à Kayes avec des machines rapportées de France. Très tôt, il s'inscrit dans une dynamique de projet économique viable et multiple. Avec les savoir-faire appris et la formation sur le tas acquise avant la migration, il met à profit le soutien financier du PDLM pour renforcer ses compétences en gestion. Ce projet de jardin lui garantit le maintien de sa double activité, d'une part au village, avec l'aide d'un membre de la famille et, d'autre part en ville, pour renforcer son activité de base, conforter son professionnalisme dans la menuiserie et développer un projet qui lui est personnel (seul le marché et le réseau de Kayes pouvant lui donner des débouchés dans la menuiserie). La diversification du projet relève d'une stratégie familiale à même de garantir la vie au village et les aspirations personnelles du promoteur.

Ce modèle de promoteur, caractérisé par le dédoublement des activités entre le village (pour la famille) et la ville (pour lui), est fréquent aussi bien à Kayes qu’à Bamako.

\section{Les échecs : des projets souvent dans l'impasse ou abandonnés}

Beaucoup de projets ont connu ou se heurtent encore à des difficultés. Sur les 96 projets de l'échantillon étudiés, 64 sont confrontés à des difficultés. Pour un tiers, les difficultés sont apparues au cours de la première année d'exercice. Un autre tiers n'a survécu que le temps d'une année avant de voir apparaître des problèmes dans le développement des activités de leurs promoteurs. Les autres projets n'ont vu les difficultés apparaître que plus tard, à partir de la troisième année d'existence. 


\section{L'impossible adaptation au contexte local} charrue, d'accessoires, d'une charrette, d'une paire de bœufs et d'un âne, constitue l'investissement de base est tributaire de la pluviométrie. Ce type de projet est actif durant la saison des pluies (3 à 4 mois) et généralement inactif en saison sèche (8 à 9 mois). Il concerne les promoteurs qui sont retournés vivre dans leur village d'origine où l'agriculture de subsistance constitue la principale activité de la population. Les migrants promoteurs d'un tel projet sont des individus sans aucune instruction scolaire. Ils ont émigré en France où ils ont généralement travaillé comme ouvrier intérimaire. Cette expérience d'un emploi temporaire (travail journalier, salaire hebdomadaire) ne leur a pas permis d'acquérir un savoir-faire qu'ils peuvent valoriser au retour par la création d'un projet de réinsertion viable. La majorité d'entre eux était en situation irrégulière et a éprouvé des difficultés pour obtenir, durant la dernière année de résidence dans le pays d'accueil, un emploi stable. À leur retour, avec peu d'argent accumulé, ils se sont installés dans leur village d'origine où ils ont retrouvé leur activité économique d'avant la migration. Ils exercent aujourd'hui une activité agricole de subsistance pour répondre aux besoins de leur famille et ont saisi l'opportunité d'une subvention du pays d'accueil (la France) pour tenter d'accroître leur production agricole. Pour eux, la réussite de leur projet s'exprime par une bonne production de mil ou de sorgho et l'autosuffisance alimentaire. Dans le cas contraire, c'est l'échec.

Les projets d'acquisition d'un véhicule de transport connaissent également d'énormes problèmes en raison des difficiles conditions d'exercice de l'activité. La région de Kayes est faiblement dotée en infrastructures routières de base. Les trajets empruntent des axes non bitumés, les pistes sont longues de plus de 100 kilomètres $^{21}$. Difficilement praticables en saison sèche, celles-ci le sont encore plus en saison des pluies. Pourtant, les migrants promoteurs investissent dans le transport et espèrent gagner de l'argent, en contribuant ainsi au désenclavement de leur village, souvent éloigné du principal centre urbain de la région qu'est la ville de Kayes. Cependant, en un an d'exercice, sur des pistes défoncées, les véhicules d'occasion ne résistent pas: les pannes se multiplient, occasionnant des frais de réparation que la modeste somme d'argent économisée durant la première année ne permet pas de couvrir. Un véhicule en bon état coûte cher dans la région de Kayes et son acquisition n'est pas à la portée des migrants qui ont vécu dans des situations difficiles à l'étranger (en situation irrégulière), qui n'ont pas accumulé d'argent (emploi non stable) et qui, au retour, ont rénové un vieux véhicule pour une activité économique exigeante. Ceux qui essaient de s'en sortir, en jonglant avec les moyens à leur disposition (épargne, achat de pièces d'occasion, etc.), vivent quotidiennement l'incertitude d'une activité très aléatoire. Les autres, constatant l'échec de leur projet, réinvestissent le capital dans un projet de substitution (magasin de céréales dans la ville de Kayes, dépôt de boisson, boutique de commerce, etc.). 

émigré est né dans une famille d'éleveurs de la région de Kayes, il ne lui est pas facile de s'engager dans un projet productif d'embouche. L'embouche ovine ne correspond pas tout fait à un élevage traditionnel : il faut savoir sélectionner des bêtes adaptées aux conditions environnementales locales, pouvoir engraisser le bétail en achetant les aliments indiqués, entreprendre l'entretien et le soin des animaux à des moments précis, établir un système de reproduction et de vente afin de dégager un profit tout en conservant au moins son capital de démarrage. Tel n'est pas le cas des promoteurs qui ont perdu une partie de leur bétail en raison de nombreux décès dus à une maladie. Ou de ceux qui n'ont pu vendre leurs bêtes parce que les races acquises n'étaient pas adaptées aux conditions climatiques locales.

L'implantation de jardins maraîchers constitue un autre exemple de l'inadaptation de certains projets au contexte environnemental local. Les jardins prometteurs sont situés au bord du fleuve Sénégal et utilisent des motopompes pour l'approvisionnement normal des plantes en eau. Pour ceux qui sont éloignés du fleuve ou d'une mare, la construction d'un puits ne remplace pas l'usage d'une motopompe, surtout dans des zones arides où les nappes d'eau sont profondes. Un projet mal localisé peut ainsi obérer définitivement le développement de l'activité et remettre en cause le mince espoir de réinsertion économique du migrant.

\section{Le poids des charges familiales}

60 Les charges familiales apparaissent comme une source de difficultés pour les projets de réinsertion ( $25 \%$ des raisons évoquées par les promoteurs). Dans un environnement hautement concurrentiel, comme l'est celui du commerce, obligeant le promoteur à faire preuve de dynamisme, d'innovation et de rationalité dans la gestion des affaires, la soustraction d'une partie des revenus pour les besoins familiaux gêne considérablement l'activité. Les magasins de commerce et les boutiques de détail, confrontés à des difficultés, sont principalement installés dans les grands marchés des centres urbains (Bamako surtout, ville de Kayes) où le potentiel de développement est très prometteur. Or, pour des projets dont les charges familiales absorbent généralement la totalité des bénéfices réalisés, parfois même plus de la moitié du capital de démarrage, il est difficile de renouveler les fonds de roulement.

61 Ainsi, un projet de commerce de moins de 3 millions de francs CFA ne dégage pas un revenu susceptible de couvrir les besoins d'une famille de plus de 10 personnes. L'individu est confronté à des dépenses familiales considérables et il n'imagine pas un instant mettre de côté de l'argent alors que sa famille a des problèmes («le projet doit faire vivre la famille»). Cela lui est d'autant plus impossible que, lors du séjour à l'étranger, les revenus de la migration étaient essentiellement consacrés à la prise en charge des dépenses de consommation de la famille restée sur place.

62 Pour les promoteurs de projet artisanal et de restauration, il en va de même. Les difficultés occasionnées par les dépenses élevées de la famille concernent des projets qui ont démarré avec un capital inférieur à 500 mille francs CFA alors que, dans le même temps, les besoins de consommation des familles des promoteurs oscillent entre 500 mille et 2 millions de francs CFA. 


\section{Difficultés de financement et de gestion : vers la faillite !}

63 Les autres explications soulevées par les promoteurs sont consécutives à des problèmes de financement (insuffisance du capital d'investissement), de gestion (ignorance des notions de base en gestion) et de maîtrise du marché (concurrence, faiblesse de la clientèle).

Les projets qui ont connu des problèmes de gestion concernent tous les secteurs. Selon les explications fournies par les promoteurs, l'abus de confiance constitue une des raisons principales de l'apparition de difficultés. C'est le cas lorsque le promoteur ne s'implique pas directement dans la gestion du projet (parce qu'il ne s'y connaît pas ${ }^{22}$ ), engage quelqu'un d'autre pour le démarrer à sa place et ne dispose pas des moyens de contrôler la façon dont son placement est géré et les activités de son projet développées. Dans ce cas, les détournements de fonds entravent le développement du projet et conduisent le plus souvent à sa faillite.

L'insuffisance du financement concerne plusieurs types de projets. Le projet de transport dont il s'agit ici n'a pas pu démarrer correctement. Le capital de départ du promoteur ( 3 millions de francs CFA) étant trop faible pour acheter un véhicule d'occasion, le rénover, le dédouaner et le mettre en circulation. Après l'avoir acquis (avec une avance de 1,8 millions de francs CFA) et rénové (1,2 millions de francs CFA), le promoteur n'a pas été en mesure de dédouaner le véhicule, alors qu'il devait encore payer, mensuellement pendant quatre mois, 300 mille francs CFA. C'est l'exemple type de promoteur qui a voulu développer une activité économique dans un secteur qu'il ne maîtrisait pas. Par la suite, le promoteur s'est reconverti comme employé dans un autre projet de transport où il occupe actuellement un poste de conducteur de véhicule.

Les deux projets de jardins maraîchers implantés au bord du fleuve Sénégal sont plutôt confrontés à des difficultés techniques, en raison de la faible capacité des installations d'approvisionnements en eau. Le niveau des financements obtenus au départ ( 2 à 3 millions de francs CFA) a permis d'acquérir des motopompes d'occasion. Au bout d'un ou de deux ans d'exercice, les machines sont devenues obsolètes, alors même que les promoteurs n'avaient pas dégagé de bénéfices pouvant leur permettre de les amortir. Selon eux, des machines modernes et de grande capacité, très chères mais nécessaires pour développer dans la région de Kayes un jardin maraîcher en milieu rural, semblent indispensables.

Pour les activités de commerce, les difficultés sont imputables à la faiblesse des bénéfices réalisés. Après déduction de la part consacrée aux frais de consommation de la famille, le reste ne permet pas la réalisation d'un réinvestissement susceptible d'augmenter le chiffre d'affaires. La croissance des activités développées est rendue impossible. Par exemple, pour le promoteur d'un magasin de friperie, ouvert avec un capital de 5 millions de francs CFA, un bénéfice annuel de 600 milles francs CFA autorise seulement l'acquisition de 10 balles de friperie, soit une quantité insignifiante pour le marché de Bamako. Le dépôt d'une caution de garantie chez le fournisseur, exigée au démarrage du projet, lui permet alors, chaque fois qu'il se trouve en rupture de stock, de s'approvisionner en marchandises, remboursables sous forme monétaire après chaque vente, sans financement complémentaire. Mais, dans ce système, les bénéfices servent à payer les dettes. Le promoteur vit alors en situation de dépendance vis-à-vis des gros investisseurs de ce secteur. Son projet a des difficultés à grandir et à lui permettre de devenir autonome après quelques années d'exercice. 
aport entre la migration et stable à l'étranger facilite pour le migrant le retour et le développement d'une activité économique dans le pays d'origine. Pour autant, le migrant qui a vécu en réseau durant toute sa migration se retrouve avec un projet individuel que les aides multiples des bailleurs de fonds et les processus de montage ont par ailleurs isolé du contexte socioéconomique local. Le migrant apparaît seul dans la difficulté alors qu'il a besoin au contraire d'un réseau pour s'en sortir (tel est le cas du secteur du commerce, activité de réseau, qui connaît le moins d'échecs). C'est à ce moment-là quand l'ancien migrant se retrouve seul, que la charge familiale devient plus lourde à supporter et contribue à anéantir le projet. Les équipements agricoles et les jardins illustrent la difficulté pour le promoteur individu de faire fructifier, tout seul, une activité : celle-ci n'est peut-être pas la plus adaptée au milieu local (en raison de l'enclavement, du manque d'eau, du problème des pièces de rechange, du manque de débouchés), mais le migrant tente «le coup ", mal conseillé, peu aidé, éloigné de tout. D'ailleurs, beaucoup d'interlocuteurs à Kayes l'ont dit durant l'enquête : s'ils avaient pu faire autre chose (mais le bailleur a insisté pour que les activités de production soient favorisées et développées dans les villages), ils auraient ouvert des boutiques et des magasins ${ }^{23}$. Les mêmes Soninkés de la région de Kayes qui se sont installés à Bamako n'ont pas hésité à se lancer dans ce qu'ils savent faire le mieux et qui rapporte le plus vite, le commerce.

décalage entre le monde de la migration et le milieu local, souvent influent, est si important que le migrant a des difficultés pour faire évoluer un projet de réinsertion économique.

\section{Conclusion}

70 Le bilan des projets est mitigé. À son retour, l'émigré promoteur se convertit difficilement en entrepreneur ${ }^{24}$. Il lui faut compter sur ses propres qualités, sur son réseau professionnel et migratoire, sur sa famille, en plus de s'appuyer sur les institutions locales et nationales. Or, les investissements publics sont trop souvent déficients pour placer dans de bonnes conditions des investisseurs aussi fragiles que les migrants. Pour se lancer et développer un projet économique, le migrant promoteur, en plus d'être motivé et formé dans cette perspective, a besoin d'infrastructures, de marchés régulés, de capitaux, d'investissements publics lourds. Le contexte devient alors surdéterminant pour le promoteur et dépasse sa seule implication: des projets pérennes demandent des réformes à tous les niveaux et une politique sociale, économique, d'aménagement, encore trop souvent peu mise en œuvre ${ }^{25}$.

71 Tout laisse à penser que certains migrants enquêtés vont par conséquent repartir à l'étranger, leur projet de réinsertion sociale et économique ayant échoué. Plus qu'un nouveau départ vers un eldorado incertain, une partie de la migration correspond au développement d'activités qui se structurent sur des réseaux transnationaux. Le commerce international est une activité qui tire facilement avantage de ce capital migratoire. À Bamako, mais aussi à Kayes où le pouvoir d'achat est élevé, les importations de produits sont croissantes, en provenance de la Côte d'Ivoire, du Sénégal, et incitent les commerçants, anciens émigrés, à investir le créneau. Il ne s'agit plus de migrations, mais d'allers et retours, temporaires, en mobilisant le réseau relationnel et en jouant constamment sur les espaces. Ces migrants deviennent alors plus hommes d'affaires qu'entrepreneurs et entretiennent, aux yeux des proches, le 
mythe de la migration enrichissante. Leur expérience bouleverse cependant quelque peu les attendus classiques de la migration : le projet initié dans le village cède le pas à des investissements urbains, moins collectifs et plus individuels, qui profitent d'abord à la personne et à sa famille. Les bailleurs de fonds doivent aussi prendre en considération ce type d'évolutions.

\section{BIBLIOGRAPHIE}

ADAMS Adrian (1977) Le long voyage des gens du fleuve, Paris, Maspéro, 170 p.

BANEGAS Richard, LOSCH Bruno (2002) La Côte d'Ivoire au bord de l'implosion, Politique Africaine, 87, octobre, pp. 139-161.

CISSE Moussa (1999) Diagnostic du territoire de la commune de Maréna Diombougou, Kayes/ Paris, GRDR, $60 \mathrm{p}$.

CONDE Julien et DIAGNE Pape Syr (1986) Les migrations internationales Sud Nord. Une étude de cas : les migrants maliens, mauritaniens et sénégalais de la vallée du fleuve Sénégal en France, Paris, OCDE, $154 \mathrm{p}$.

DAUM Christophe (1998) Les associations de Maliens en France. Migrations, développement et citoyenneté, Paris, Karthala, 253 p.

MINISTERE DE L'ÉCONOMIE, DES FINANCES ET DU PLAN (1994) Enquête Malienne de Conjoncture Economique et sociale, Bamako, Direction Nationale de la Statistique et de l'Informatique (DNSI).

DULAU Caroline (2003) Commerce et communication dans une ville enclavée du Mali, NETCOM, 17, 1-2, pp. 113-118.

GUBERT Flore (1996) Transferts des travailleurs émigrés, facteurs de réduction de la pauvreté au Mali, Communication au colloque Systèmes et dynamiques des migrations internationales ouest africaines, Dakar, 3-6 décembre.

IGUE John (1989) Le développement des périphéries nationales en Afrique, in B. Antheaume, C. Blanc-Pamard et alii, Tropiques, lieux et liens, Paris, ORSTOM, pp. 594-605.

KANE F. et LERICOLLAIS A. (1975) L'émigration des Soninké, Cahiers ORSTOM, Série Sciences Humaines, XII, 2, pp. 177-187.

LOMBARD Jérôme (1993) Riz des villes, mil des champs en pays Serer - Sénégal, Talence, CEGET, $226 \mathrm{p}$.

LOMBARD Jérôme (2002) Mali : faire une nation avant l'intégration régionale ? L'Espace Géographique, 3, pp. 276-279.

LOMBARD Jérôme et NINOT Olivier (2002) Impasses et défis dans le transport routier, in Momar Coumba Diop, La société sénégalaise entre le local et le global, Paris, Karthala, pp. 109-162.

PAPAIL Jean (2002) Migrations internationales, transferts monétaires et investissements dans les milieux urbains du centre-ouest mexicain, Autrepart, 23, pp. 89-105.

POLITIQUE AFRICAINE, n56, 1994, décembre, Entrepreneurs - Ajustement et démocratie. 
QUIMINAL Catherine (1991), Gens d'ici, gens d'ailleurs - Migrations soninké et transformations villageoises, Paris, Bourgois.

REMUAO (1996) Enquête Malienne sur les Migrations et l'Urbanisation (EMMU-1993), Bamako.

REMUAO (1998) Synthèses régionales, Études et travaux du CEPED, 14, juin et 15, octobre.

SOUMARE Siré (2001) Après l'émigration le retour à la terre, Bamako, Editions Jamana, 166 p.

TARRIUS Alain (1991) Les fourmis de l'Europe, Arcueil, INRETS, rapport n 140, 210 p.

VOLBRECHT Laëtitia (1999) Le rôle des transports de marchandises à Kayes au Mali, Université de Paris I - La Sorbonne, Institut de Géographie, mémoire de maîtrise, 103 p.

\section{NOTES}

1. C'est le cas au Mali, notamment dans la région de Kayes, où un retour collectif de migrants en provenance de la France a été organisé en 1976 en vue de la réinsertion autour d'un projet collectif agricole. Cf. Soumaré, 2001.

2. L'échantillon se décompose ainsi : une cinquantaine de migrants ont été interrogés dans la région de Kayes, l'autre moitié à Bamako.

3. Le recensement général de la population et de l'habitat de 1998 a permis de dénombrer un effectif de 42000 migrants internationaux rentrés entre 1993 et 1997.

4. Burkina Faso, Côte d'Ivoire, Guinée, Mali, Mauritanie, Niger et Sénégal.

5. La grave crise politique qui a éclaté en septembre 2002 en Côte d'Ivoire risque d'entraîner un reflux important des ressortissants maliens installés dans ce pays dont les conséquences en termes de réinsertion sociale et économique dans le pays d'origine sont imprévisibles. Pour un aperçu de la crise, cf. Banégas et Losch, 2002.

6. Le lecteur se référera aux travaux de John Igue sur les périphéries nationales. Cf. Igue, 1989.

7. Par exemple, la Côte d'Ivoire a instauré la carte de séjour obligatoire aux étrangers, appliquée notamment aux ressortissants des pays membres de la CEDEAO.

8. Bénin, Burkina Faso, Côte d'Ivoire, Cap-Vert, Guinée, Guinée-Bissau, Ghana, Liberia, Mali, Mauritanie, Niger, Nigeria, Sénégal, Sierra Leone, Togo.

9. C'est le cas pour les bénéficiaires du Programme de Développement Local et Migration (PDLM) : selon les statistiques de l'Office des Migrations Internationales (OMI), les 404 projets de réinsertion, financés par la France entre 1996 et 2000, ont essentiellement été développés à Bamako et, dans une moindre mesure, à Kayes.

10. Cf. Gubert, $1996:$ p. 8

11. L'enquête a eu lieu en 2002 et porte sur des projets démarrés, pour les plus anciens, en 1996.

12. Une étude de 1999, réalisée dans la ville de Kayes, montrait l'importance de l'activité de transport routier dans cette région, pourtant dépourvue de bonnes infrastructures. Cf. Volbrecht, 1999.

13. Le boom des télécommunications est réel dans la ville de Kayes et à Bamako. Comme dans le transport, il n'est pas à la portée de tout le monde. Cf. Dulau, 2003.

14. Programme initié par la coopération française.

15. La comparaison avec d'autres zones migratoires, notamment entre Mexique et États-Unis, indique une préférence des émigrés pour les retours productifs en ville qui leur permettent de devenir chefs de petites entreprises ou travailleurs indépendants. Cf. Papail, 2002.

16. Dans les années 1985-1986, au Sénégal, Jérôme Lombard a montré que, même en période de sécheresse et de crise agricole, les grandes concessions familiales produisaient beaucoup plus de 
vivres que les petites, notamment en raison d'une gestion de la main-d'œuvre sur l'année plus aisée. Cf. Lombard, 1993.

17. Ce chiffre est à rapprocher des $59 \%$ d'émigrés enquêtés qui ont déclaré n'avoir aucune instruction scolaire.

18. Pour certains enquêtés, il est difficile de savoir si le retour a fait suite à un rapatriement forcé ou si c'est la fin du voyage (la «fatigue " selon leurs propres termes) qui les a poussés à rentrer. La fierté de ne pas révéler la vérité ou la honte d'avoir été expulsé l'emportant sur toute autre considération.

19. La variable subvention est discriminante dans la réussite ou l'échec des projets. $49 \%$ des projets de l'échantillon ont été subventionnés par le pays d'accueil (la France) et $51 \%$ des projets (qui concernent les promoteurs revenus d'Afrique) n'ont rien reçu. S'il y a autant de réussites chez les migrants subventionnés que chez les migrants n'ayant reçu aucune subvention, le pourcentage d'échec est plus élevé chez les migrants de retour bénéficiaires d'aide à la réinsertion économique ( $28 \%$ ) que chez ceux réinstallés sans aide (20\%).

20. Dans un sous-échantillon de 161 personnes, bénéficiaires des programmes d'aide au retour à Bamako (25) et à Kayes (136), 20 individus sont repartis à l'étranger, soit $12 \%$.

21. Autour de la ville de Kayes, l'amélioration du réseau et son bitumage sont en cours. Cf. Lombard, 2002.

22. Dans le transport, c'est très fréquent. Cf. Lombard et Ninot, 2002.

23. Adrian Adams rappelle combien la population soninké est de longue date portée sur le commerce. Cf. Adams, 1977.

24. Cf. le numéro 56 de Politique Africaine consacré aux entrepreneurs.

25. Pourtant, la décentralisation malienne est remarquable et très avancée: elle devrait permettre, à l'échelle des communes urbaines et rurales, de mieux soutenir les migrants promoteurs de projets. Par exemple, la commune rurale de Maréna Diombougou (région de Kayes), dont le Maire est un ancien immigré en France, a élaboré un document de diagnostic du territoire de la commune dans lequel le migrant est intégré comme co-initiateur et acteur de financement des projets communaux. Cf. Cissé, 1999.

\section{INDEX}

Index géographique : Mali

Mots-clés : aide au développement, investissements, réinsertion au pays d'origine, retour

\section{AUTEURS}

\section{BABACAR NDIONE}

Consultant, Sénégal, Adresse de courrier : S/C de Richard Lalou, IRD, B.P. 1386, Dakar-Hann. Sénégal. Tél. : (221) 644.97.46. E-mail : bndione2002@hotmail.com

\section{JÉRÔME LOMBARD}

Chargé de recherches, IRD, B.P. 1386, Dakar-Hann. Sénégal, Tél. : (221) 865 19.12, Lombard@ird.sn 